\title{
THE FINITE-SECTION APPROXIMATION FOR INTEGRAL EQUATIONS ON THE HALF-LINE
}

\author{
FRANK DE HOOG' ${ }^{1}$ AND IAN H. SLOAN ${ }^{2}$
}

(Received 20 September 1985; revised 21 February 1986)

\begin{abstract}
Integral equations on the half line are commonly approximated by the finite-section approximation, in which the infinite upper limit is replaced by a positive number $\beta$. A novel technique is used here to rederive a number of classical results on the existence and uniqueness of the solution of the Wiener-Hopf and related equations, and is then extended to obtain existence, uniqueness and convergence results for the corresponding finite-section equations. Unlike the methods used in the recent work of Anselone and Sloan, the present methods are constructive, and result in explicit asymptotic bounds for the error introduced by the finite-section approximation.
\end{abstract}

\section{Introduction.}

In this paper, we consider integral equations of the form

$$
y(t)-\int_{0}^{\infty}[w(t-s)+h(t, s)] y(s) d s=f(t), \quad t \geqslant 0,
$$

where $w \in L_{1}(\mathbb{R}), h$ satisfies the conditions indicated below, and $f, y \in$ $L_{\infty}^{+}:=L_{\infty}\left(\mathbb{R}^{+}\right)$. Our main concern is the approximation of this equation by the corresponding 'finite-section' equation

$$
y_{\beta}(t)-\int_{0}^{\beta}[w(t-s)+h(t, s)] y_{\beta}(s) d s=f(t), \quad 0 \leqslant t \leqslant \beta,
$$

in which the upper limit of integration is replaced by a positive number $\beta$.

\footnotetext{
${ }^{1}$ DMS, C.S.I.R.O., P.O. Box 1965, Canberra, A.C.T. 2601, Australia

${ }^{2}$ Department of Applied Mathematics, University of New South Wales, Sydney, N.S.W. 2033, Australia

○ Copyright Australian Mathematical Society 1987, Serial-fee code 0334-2700/87
} 
The conditions to be satisfied by $h$ are

$$
\begin{gathered}
\sup _{t \geqslant 0} \int_{0}^{\infty}|h(t, s)| d s<\infty, \\
\lim _{t^{\prime} \rightarrow t} \int_{0}^{\infty}\left|h\left(t^{\prime}, s\right)-h(t, s)\right| d s=0, \quad 0 \leqslant t<\infty, \\
\lim _{t \rightarrow \infty} \int_{0}^{\infty}|h(t, s)| d s=0 .
\end{gathered}
$$

An important special case is $h(t, s) \equiv 0$, in which case (1.1) is a Wiener-Hopf equation.

We may conveniently write $(1.1)$ as

$$
\left(I^{+}-W^{+}-H^{+}\right) y=f
$$

where $I^{+}$is the identity operator for functions defined on $\mathbb{R}^{+}$, and $W^{+}$and $H^{+}$ are integral operators on $\mathbb{R}^{+}$with kernels $w(t-s)$ and $h(t, s)$ respectively. The Wiener-Hopf operator $W^{+}$is a bounded operator on $L_{\infty}^{+}$, and the operator $H^{+}$is a compact operator on $L_{\infty}^{+}$(see for example Atkinson [2]).

In a recent paper by Anselone and Sloan [1], the convergence of $y_{\beta}(t)$ to $y(t)$, uniformly for $t$ on an arbitrary finite interval, was established under suitable conditions. The proofs used in that work were non-constructive, being based on a repeated use of the Arzelà-Ascoli theorem, and so provide no foundation for the estimation of errors. In this paper we rederive many of the results of Anselone and Sloan [1] by constructive arguments, and then go on to study the nature of the approximation of $y(t)$ by $y_{\beta}(t)$ (see Theorem 5.2).

A key result obtained in [1] is that the solution operator for (1.2) (or more precisely, for that equation amended by allowing $t$ to run over $\mathbb{R}^{+}$, and then viewed in the space $X^{+}$of bounded, continuous functions on $\mathbb{R}^{+}$) exists and is uniformly bounded for all $\beta$ sufficiently large. Results of this kind are useful not only for establishing the convergence of $y_{\beta}(t)$ to $y(t)$, but also, as in a recent paper by Sloan and Spence [6], for establishing convergence and error bounds for numerical methods. In the present work the existence and uniform boundedness of the solution operator for the equation (1.2) is established in an appropriate space (namely $L_{\infty}(0, \beta)$ ), with the important difference that an explicit bound on the norm of the solution operator is now obtained (see (4.8) and (4.9)).

\section{The Wiener-Hopf equation}

Before we can attempt to answer questions about the existence and uniqueness of solutions of the finite-section equation (1.2), we require similar results for the Wiener-Hopf equation. In this section we derive these and other related results in a somewhat novel way. It should be said, however, that none of the results in this 
section are new. Moreover, not all of the known results for the Wiener-Hopf equation can be obtained in this manner (see Krein [5] for a full treatment of the Wiener-Hopf equation). Nevertheless, the techniques used are of interest in their own right and, more importantly, can be extended to analyse the finite-section equation.

The Wiener-Hopf equation, that is (1.1) with $h(t, s)$ set equal to zero, is written here as

$$
y^{+}(t)-\int_{0}^{\infty} w(t-s) y^{+}(s) d s=f^{+}(t), \quad t>0
$$

or

$$
\left(I^{+}-W^{+}\right) y^{+}=f^{+} \text {. }
$$

It turns out to be convenient to study also the corresponding equation on the negative real axis,

$$
y^{-}(t)-\int_{-\infty}^{0} w(t-s) y^{-}(s) d s=f^{-}(t), \quad t<0,
$$

or

$$
\left(I^{-}-W^{-}\right) y^{-}=f^{-} \text {. }
$$

These equations may be considered in any of the spaces $L_{p}^{ \pm}:=L_{p}\left(\mathbb{R}^{ \pm}\right)$, $1 \leqslant p \leqslant \infty$. Also, it is sometimes convenient to work in the Banach space $Z^{ \pm}:=L_{1}^{ \pm} \cup L_{\infty}^{ \pm}$, equipped with the norm

$$
\|\cdot\|_{Z^{ \pm}}=\|\cdot\|_{L_{1}^{ \pm}}+\|\cdot\|_{L_{\infty}^{ \pm}} .
$$

It may be easily shown that $Z^{ \pm} \subset L_{p}^{ \pm}, 1 \leqslant p \leqslant \infty$.

Now let $E^{ \pm}$, respectively, denote any of the spaces $Z^{ \pm}, L_{p}^{ \pm}, 1 \leqslant p \leqslant \infty$. Then it is known that $W^{ \pm}$is a bounded operator on $E^{ \pm}$, with norm satisfying

$$
\left\|W^{ \pm}\right\|_{E^{ \pm}} \leqslant \int_{-\infty}^{+\infty}|w(s)| d s=\|w\|_{L_{1}} .
$$

(For the case $E^{ \pm}=L_{p}^{ \pm}$see, for example, [5, Lemma 6.1]. The result for the case $E^{ \pm}=Z^{ \pm}$then follows trivially.)

It may be remarked that in equations (2.1) and (2.2), and similar equations throughout the paper, the solutions are not defined at $t=0$. This is merely a matter of convenience, the values at the single point $t=0$ being of no consequence. Throughout the paper we shall use the convention that equations need not hold at the special point $t=0$.

With that understanding, we shall see that equations (2.1) and (2.2), taken together, may be viewed as a single integral equation on the whole line (see (2.8)). The central idea of our analysis is to view that equation as a perturbation of the convolution equation

$$
y(t)-\int_{-\infty}^{\infty} w(t-s) y(s) d s=f(t), \quad-\infty<t<\infty,
$$


or

$$
(I-W) y=f,
$$

for which the analysis is relatively straightforward. The latter equation can be considered on any of the Banach spaces $L_{p}:=L_{p}(\mathbb{R}), 1 \leqslant p \leqslant \infty$, or on $Z:=L_{1}$ $\cap L_{\infty}$, equipped with the norm

$$
\|\cdot\|_{Z}=\|\cdot\|_{L_{1}}+\|\cdot\|_{L_{\infty}}
$$

It can easily be seen that $Z \subset L_{p}$ and $\|g\|_{L_{p}} \leqslant\|g\|_{Z}$ for $1 \leqslant p \leqslant \infty$, and in analogy with (2.4) we have

$$
\|W\|_{E} \leqslant\|w\|_{L_{1}}
$$

where $E$ denotes any of the spaces $Z, L_{p}, 1 \leqslant p \leqslant \infty$.

The invertibility properties of (2.5) are known to depend on the set of values taken by the Fourier transform

$$
\hat{w}(\nu)=\int_{-\infty}^{\infty} w(s) \exp (i \nu s) d s, \quad-\infty<\nu<\infty .
$$

Specifically, Wiener [7] has shown that:

Lemma 2.1. The operator $I-W$ has a bounded inverse on the space $L_{1}$ if and only if

$$
1-\hat{w}(\nu) \neq 0, \quad-\infty<\nu<\infty .
$$

Furthermore, if (2.7) holds then the inverse on the space $L_{1}$ is

$$
(I-W)^{-1}=I+V,
$$

where $V$ is an integral operator with kernel $v(t-s)$, and $v \in L_{1}$ is such that

$$
(1-\hat{w}(\nu))^{-1}=1+\hat{v}(\nu), \quad-\infty<\nu<\infty .
$$

For the more general spaces described above, the following corollary of Lemma 2.1 is well known.

Corollary 2.2. Let the condition (2.7) hold, and let $E$ be any of the spaces $Z$, $L_{p}, 1 \leqslant p \leqslant \infty$. Then $(I-W)$ has a bounded inverse on $E$, and

$$
(I-W)^{-1}=I+V
$$

where $V$ is the integral operator described in Lemma 2.1 .

We shall now use the above results for the integral equation on the whole line to deduce the existence of solutions of the Wiener-Hopf equation on the half line, under appropriate conditions. The first step is to recognize that the two equations (2.1) and (2.2) may be viewed as a single integral equation on the real line, with a 
discontinuous kernel. If $y$ and $f$ are defined by

$$
y(t)= \begin{cases}y^{+}(t), & t>0 \\ y^{-}(t), & t<0\end{cases}
$$

and

$$
f(t)= \begin{cases}f^{+}(t), & t>0, \\ f^{-}(t), & t<0,\end{cases}
$$

then (2.1) and (2.2) taken together are equivalent to

$$
(I-W+A) y=f,
$$

where $A$ is the integral operator on $\mathbb{R}$ with kernel $a(t, s)$ given by

$$
a(t, s)= \begin{cases}0, & t s>0, \\ w(t-s), & t s<0 .\end{cases}
$$

We now show that the operator $A$ is compact.

LEMMA 2.3. The operator $A$ is compact on $E$, where $E$ is any of the spaces $Z, L_{p}$, $1 \leqslant p \leqslant \infty$.

Proof. We first show that $A$ is a compact operator on $L_{\infty}$; more precisely, it is a compact operator from $L_{\infty}$ to the closed subspace $D_{0}$, consisting of functions that are continuous on $\mathbb{R}^{+}$and $\mathbb{R}^{-}$, vanish at infinity, and have a simple discontinuity at 0 .

For this purpose it is convenient to write

$$
A=A_{1}+A_{2}
$$

where

$$
\left(A_{1} g\right)(t)= \begin{cases}\int_{-\infty}^{0} w(t-s) g(s) d s, & t>0 \\ 0 & t<0\end{cases}
$$

and

$$
\left(A_{2} g\right)(t)= \begin{cases}0, & t>0 \\ \int_{0}^{\infty} w(t-s) g(s) d s, & t<0 .\end{cases}
$$

It is clear that the family

$$
\left\{A_{1} g: g \in L_{\infty},\|g\|_{L_{\infty}} \leqslant 1\right\}
$$

is uniformly equicontinuous on $(0, \infty)$, and is equiconvergent to zero at $\infty$. Since each member of the family vanishes on $(-\infty, 0)$, it then follows from a simple adaptation of the Arzelà-Ascoli theorem, in the manner of Atkinson [2], that $A_{1}$ is compact as an operator from $L_{\infty}$ to $D_{0}$. A similar argument shows that $A_{2}$ has the same property, and hence that $A$ is compact from $L_{\infty}$ to $D_{0}$. 
An almost identical argument shows that the adjoint integral operator $A^{*}$ (i.e. the integral operator with the conjugate transpose kernel $\overline{a(s, t)}$ ) is a compact operator from $L_{\infty}$ to $D_{0}$, from which it follows, by a standard argument, that $A$ is a compact operator on the space $L_{1}$.

Since $A$ is compact as an operator on both $L_{\infty}$ and $L_{1}$, it follows easily that $A$ is compact on $Z=L_{1} \cap L_{\infty}$. More profoundly, since $A$ is compact as an operator from $L_{\infty}$ to $D_{0}$ and is also bounded on $L_{1}$, it follows from an interpolation result of Krasnosel'skii [4, theorem 2] that $A$ is compact as an operator on $L_{p}$ for each $p$ in $1<p<\infty$. (A trivial modification of the argument in [4] accommodates the discontinuity at 0 .) Thus the result is proved.

We now exploit the compactness property established in Lemma 2.3. Let $E$ denote any of the spaces $Z, L_{p}, 1 \leqslant p \leqslant \infty$, and assume that the condition (2.7) holds. Then by Corollary (2.2) $I-W$ has a bounded inverse on $E$, and so, trivially, is a Fredholm operator of index zero. (For the definition of a Fredholm operator see, for example, Jörgens [3, §5.3].) Since $A$ is compact on $E$, it follows that the operator $I-W+A$ is also a Fredholm operator of index zero, because the addition of a compact operator to a Fredholm operator yields another Fredholm operator of the same index (see, for example, [3, theorem 5.12]). The following result is then obtained by an application of the Fredholm alternative [3, theorem 5.17], in a special setting.

TheOREM 2.4. Let condition (2.7) hold, and let $E$ be any of the spaces $Z, L_{p}$, $1 \leqslant p \leqslant \infty$.

(i) The equation

$$
(I-W+A) x=0, \quad x \in E,
$$

has a finite number of linearly independent solutions. Each such solution $x$ belongs to $Z$, and hence also to $L_{p}, 1 \leqslant p \leqslant \infty$.

(ii) The equation

$$
(I-W+A) y=f, \quad f \in E,
$$

has a solution $y \in E$ if and only if $f$ satisfies

$$
\int_{-\infty}^{\infty} f(s) x(-s) d s=0
$$

for every solution of

$$
(I-W+A) x=0, \quad x \in Z .
$$

(iii) Equation (2.10) has a solution $y \in E$ for all $f \in E$ if and only if (2.11) has only the trivial solution.

Proof. The first result, that (2.9) has a finite number of linearly independent solutions, follows from the fact that $I-W+A$ is a Fredholm operator. We denote the number of linearly independent solutions of (2.9) by $n(E)$. 
The remaining results are obtained by applying the Fredholm theory in the setting of the 'dual system' formed by the Banach space pair $E, Z$, with the associated bounded bilinear form

$$
\langle g, x\rangle=\int_{-\infty}^{\infty} g(s) x(s) d s, \quad g \in E, x \in Z .
$$

(For a discussion of dual systems see, for example, [3, §3.5].) The properties required for $(E, Z)$ to be a dual system, namely

$$
\begin{aligned}
& \left\langle g_{0}, x\right\rangle=0 \text { for some } g_{0} \in E \text { and all } x \in Z \Rightarrow g_{0}=0, \\
& \left\langle g, x_{0}\right\rangle=0 \text { for some } x_{0} \in Z \text { and all } g \in E \Rightarrow x_{0}=0,
\end{aligned}
$$

are easily established.

With respect to this dual system the operator $J:=I-W+A$ has a transpose $J^{T}$, defined by

$$
\langle J g, x\rangle=\left\langle g, J^{T} x\right\rangle, \quad g \in E, x \in Z .
$$

The transpose is given explicitly by

$$
J^{T}=(I-W+A)^{T}=I-W^{T}+A^{T},
$$

where $W^{T}$ and $A^{T}$ are the integral operators on $\mathbb{R}$ with the transposed kernels $w^{T}(t, s)=w(s-t)$ and

$$
a^{T}(t, s)=a(s, t)= \begin{cases}0, & t s>0, \\ w(s-t), & t s<0 .\end{cases}
$$

Since $W^{T}$ and $A^{T}$ can be obtained from $W$ and $A$ merely by replacing $w$ by $\tilde{w}$, where $\tilde{w}(s)=w(-s)$, it follows by the argument used previously that $J^{T}$ is a Fredholm operator of index zero, operating on the Banach space $Z$.

Under these circumstances, the Fredholm alternative [3, theorem 5.17] can be applied to $I-W+A$ and its transpose. The first consequence is that the equation

$$
\left(I-W^{T}+A^{T}\right) \tilde{x}=0, \quad \tilde{x} \in Z,
$$

has the same number of linearly independent solutions as (2.9), namely $n(E)$. But $\left(2.11^{\prime}\right)$ is equivalent, under the transformation $\tilde{x}(s)=x(-s)$, to $(2.11)$, thus we conclude that (2.9) and (2.11) have the same number of linearly independent solutions, or $n(E)=n(Z)$. It follows (since $Z \subset E$ ) that the solution sets of (2.9) and (2.11) coincide, so proving part (i) of the theorem.

The Fredholm alternative [3, theorem 5.17] also requires that $(2.10)$ has a solution $y \in E$ if and only if

$$
\langle f, \tilde{x}\rangle=0
$$

for every solution $\tilde{x}$ of $\left(2.11^{\prime}\right)$. Under the transformation $\tilde{x}(s)=x(-s)$, the latter statement is equivalent to that in part (ii) of the theorem. Thus (ii) is proved. For the solution to exist for all $f \in E$ one requires (2.12) to hold for all $f \in E$, 
implying $\tilde{x}=0$, and hence $x=0$ for every solution of (2.11). Conversely, if (2.11) has only the trivial solution then, by (ii), (2.10) has a solution for all $f \in E$. Thus (iii) holds.

We may now use the foregoing theorem to obtain analogous results for the Wiener-Hopf equation:

THEOREM 2.5. Let condition (2.7) hold, and let $E^{+}$denote any of the spaces $Z^{+}$, $L_{p}^{+}, 1 \leqslant p \leqslant \infty$, and $E^{-}$the corresponding space $Z^{-}$or $L_{p}^{-}$.

(i) The equation

$$
\left(I^{ \pm}-W^{ \pm}\right) x^{ \pm}=0, \quad x^{ \pm} \in E^{ \pm},
$$

has a finite number of linearly independent solutions. Each such solution $x^{ \pm}$belongs to $Z^{ \pm}$, and hence also to $L_{p}^{ \pm}, 1 \leqslant p \leqslant \infty$.

(ii) The equation

$$
\left(I^{+}-W^{+}\right) y^{+}=f^{+}, \quad f^{+} \in E^{+},
$$

has a solution $y^{+} \in E^{+}$if and only if

$$
\int_{0}^{\infty} f^{+}(s) x^{-}(-s) d s=0
$$

for every solution $x^{-}$of

$$
\left(I^{-}-W^{-}\right) x^{-}=0, \quad x^{-} \in Z^{-} .
$$

The analogous result also holds if + and - are interchanged.

(iii) Equation (2.14) has a solution $y^{+} \in E^{+}$for all $f^{+} \in E^{+}$if and only if (2.15) has only the trivial solution. A similar result holds if + and - are interchanged.

Proof. The two versions $(+$ and -$)$ of $(2.13)$ are equivalent, taken together, to

$$
(I-W+A) x=0, \quad x \in E,
$$

if $E$ is the space corresponding to $E^{ \pm}$for functions defined on the whole line, and if $x$ is related to $x^{ \pm}$by

$$
x(t)= \begin{cases}x^{+}(t), & t>0 \\ x^{-}(t), & t<0 .\end{cases}
$$

Part (i) of the theorem therefore follows from Theorem 2.4 part (i).

In a similar way, the pair of equations

$$
\left(I^{+}-W^{+}\right) y^{+}=f^{+}, \quad y^{+}, f^{+} \in E^{+},
$$

and

$$
\left(I^{-}-W^{-}\right) y^{-}=0, \quad y^{-} \in E^{-},
$$

is equivalent to

$$
(I-W+A) y=f, \quad y, f \in E,
$$


provided

$$
f(t)= \begin{cases}f^{+}(t), & t>0 \\ 0, & t<0\end{cases}
$$

and

$$
y(t)= \begin{cases}y^{+}(t), & t>0 \\ y^{-}(t), & t<0\end{cases}
$$

It now follows from Theorem 2.4 part (ii) that equation (2.16) has a solution $y \in E$ (and hence (2.14) has a solution $y^{+} \in E^{+}$) if and only if

$$
\int_{0}^{\infty} f^{+}(s) x^{-}(-s) d s=0
$$

for every solution $x^{-}$of (2.15). Thus (ii) holds. If (2.14) holds for all $f^{+} \in E^{+}$then (2.17) must hold for all $f^{+} \in E^{+}$, from which it follows that $x^{-}=0$, so that (2.15) has only the trivial solution. Conversely, if (2.15) has only the trivial solution, then, by (ii), (2.14) has a solution for each $f^{+} \in E^{+}$, so proving (iii).

We can now deduce a number of equivalent conditions for ensuring that the Wiener-Hopf equation (2.1) and the associated equation on the real line (2.8) have unique solutions for arbitrarily chosen right-hand sides.

THEOREM 2.6. Let condition (2.7) hold. Then the following are equivalent.

(a) $I-W+A$ has a bounded inverse on $L_{p}$ for all $p$ satisfying $1 \leqslant p \leqslant \infty$.

(b) $I-W+A$ has a bounded inverse on $L_{p}$ for some $p$ satisfying $1 \leqslant p \leqslant \infty$.

(c) $I-W+A$ has a bounded inverse on $Z$.

(d) The equation $(I-W+A) x=0$ has no nontrivial solution $x \in Z$.

(e) $I^{+}-W^{+}$and $I^{-}-W^{-}$have bounded inverses on $L_{p}^{+}$and $L_{p}^{-}$respectively for all $p$ satisfying $1 \leqslant p \leqslant \infty$.

(f $\left.{ }^{ \pm}\right) I^{ \pm}-W^{ \pm}$has a bounded inverse on $L_{p}^{ \pm}$for some $p$ satisfying $1 \leqslant p \leqslant \infty$.

$\left(\mathrm{g}^{ \pm}\right) I^{ \pm}-W^{ \pm}$has a bounded inverse on $Z^{ \pm}$.

(h) Neither $\left(I^{+}-W^{+}\right) x^{+}=0$ nor $\left(I^{-}-W^{-}\right) x^{-}=0$ has a nontrivial solution $x^{ \pm} \in Z^{ \pm}$.

(Note that $\left(\mathrm{f}^{+}\right)$and $\left(\mathrm{f}^{-}\right)$, and similarly $\left(\mathrm{g}^{+}\right)$and $\left(\mathrm{g}^{-}\right)$, are to be considered as separate equivalent conditions in the theorem.)

Proof. The implications (a) $\Rightarrow$ (b) $\Rightarrow$ (d) and (c) $\Rightarrow$ (d) are trivial, and the implications (d) $\Rightarrow$ (a) and (d) $\Rightarrow$ (c) follow from Theorem 2.4. Thus (a), (b), (c), (d) are equivalent. 
The implications $(\mathrm{e}) \Rightarrow\left(\mathrm{f}^{ \pm}\right)$are trivial, and the implications $\left(\mathrm{f}^{ \pm}\right) \Rightarrow(\mathrm{h})$ and $\left(\mathrm{g}^{ \pm}\right) \Rightarrow(\mathrm{h})$ follow from Theorem 2.5 , as also do $(\mathrm{h}) \Rightarrow(\mathrm{e})$ and $(\mathrm{h}) \Rightarrow\left(\mathrm{g}^{ \pm}\right)$. Thus (e), $\left(f^{ \pm}\right),\left(g^{ \pm}\right),(h)$ are equivalent.

Finally, (d) and (h) are equivalent under the correspondence $x^{ \pm}=\left.x\right|_{R^{ \pm}}$.

\section{Modified Wiener-Hopf equation.}

In this section we return to the full equation on the half-line, equation (1.1), which we now write as

$$
\left(I^{+}-W^{+}-H^{+}\right) y^{+}=f^{+} .
$$

Since $H^{+}$is a compact operator on the space $L_{\infty}^{+}$(see section 1 ), it will be convenient to consider this equation in the space $L_{\infty}^{+}$.

As in Section 2, we shall also consider an equation on the negative real axis,

$$
\left(I^{-}-W^{-}\right) y^{-}=f^{-} \text {. }
$$

Equations (3.1) and (3.2) can then be viewed as a single equation on the whole real line, namely

$$
(I-W+A-H) y=f,
$$

where $W$ and $A$ are as in section 2, and $H$ is an integral operator with kernel $h(t, s)$ for $t, s>0$, and zero otherwise.

THEOREM 3.1. Let condition (2.7) hold, and suppose that

$$
(I-W+A-H) x=0, \quad x \in L_{\infty},
$$

has no nontrivial solution $x$. Then $I-W+A-H$ has a bounded inverse on $L_{\infty}$, and $I-W+A$ has a bounded inverse on $L_{p}, 1 \leqslant p \leqslant \infty$.

Proof. Since $H^{+}$is compact on $L_{\infty}^{+}$, it follows easily that $H$ is compact on $L_{\infty}$. Thus $I-W+A-H$ as an operator on $L_{\infty}$ is a Fredholm operator with index zero. Since the homogeneous equation (3.4) has only the trivial solution, it follows from the Fredholm alternative that $I-W+A-H$ has a bounded inverse on $L_{\infty}$.

It then follows from (3.3), on restricting attention to the negative real axis, that (3.2) has a unique solution $y^{-} \in L_{\infty}^{-}$for each $f^{-} \in L_{\infty}^{-}$, and hence that $I^{-}-W^{-}$ has a bounded inverse on $L_{\infty}^{-}$. The remaining result, that $I-W+A$ has a bounded inverse on $L_{p}$ for $1 \leqslant p \leqslant \infty$, now follows from Theorem 2.6 (a) and $\left(f^{-}\right)$.

We may also state an equivalent result for operators directly relevant to the half-line. The result is similar to one obtained by Anselone and Sloan [1, Theorem 9.3]. 
THEOREM 3.2. Let condition (2.7) hold, and suppose that neither

$$
\left(I^{+}-W^{+}-H^{+}\right) x^{+}=0, \quad x^{+} \in L_{\infty}^{+},
$$

nor

$$
\left(I^{-}-W^{-}\right) x^{-}=0, \quad x^{-} \in L_{\infty}^{-},
$$

has a nontrivial solution. Then $I^{+}-W^{+}-H^{+}$has a bounded inverse on $L_{\infty}^{+}$, and $I^{ \pm}-W^{ \pm}$have bounded inverses on $L_{p}^{ \pm}, 1 \leqslant p \leqslant \infty$.

\section{The finite-section equation}

In this section we show that a unique solution of the finite-section equation (1.2) exists when $\beta$ is sufficiently large.

Our approach to the finite-section equation is simliar to that in the previous sections. We begin by incorporating (1.2) into an equation on the whole line. Specifically, we consider the three equations

$$
\begin{aligned}
& y(t)-\int_{\beta}^{\infty} w(t-s) y(s) d s=f(t), \quad t>\beta, \\
& y(t)-\int_{0}^{\beta} w(t-s) y(s) d s-\int_{0}^{\beta} h(t, s) y(s) d s=f(t), \quad 0<t<\beta, \\
& y(t)-\int_{-\infty}^{0} w(t-s) y(s) d s=f(t), \quad t<0,
\end{aligned}
$$

as a single integral equation over the whole line, with a discontinuous kernel. This equation can be written as

$$
\left(I-W+A-H+A_{\beta}-\Delta_{\beta}\right) y=f,
$$

where $W, A$ and $H$ are as defined previously, and $A_{\beta}, \Delta_{\beta}$ are defined by

$$
\left(A_{\beta} y\right)(t)= \begin{cases}\int_{-\infty}^{\beta} w(t-s) y(s) d s, & t>\beta, \\ \int_{\beta}^{\infty} w(t-s) y(s) d s, & t<\beta,\end{cases}
$$

and

$$
\left(\Delta_{\beta} y\right)(t)= \begin{cases}\int_{-\infty}^{0} w(t-s) y(s) d s-\int_{0}^{\infty} h(t, s) y(s) d s, \quad t>\beta \\ -\int_{\beta}^{\infty} h(t, s) y(s) d s, & 0<t<\beta, \\ \int_{\beta}^{\infty} w(t-s) y(s) d s, & t<0 .\end{cases}
$$


Note that $A_{\beta}$ is related to $A$ by a translation: if $y_{\beta}^{\prime}(t)=y(t-\beta)$, then

$$
\left(A_{\beta} y_{\beta}^{\prime}\right)(t)=(A y)(t-\beta) \text {. }
$$

THEOREM 4.1. Assume that condition (2.7) holds, and that

$$
(I-W+A-H) x=0, x \in L_{\infty},
$$

nas oniy ine iriviai soiulion. Then for $\beta$ sufficienily large $I-W+A-H+A_{\beta}-$ $\Delta_{\beta}$ has a bounded inverse on $L_{\infty}$. Moreover, there exists a constant $c$, independent of $\beta$ and $f$, such that the solution of (4.2) satisfies

$$
\|y\|_{L_{\infty}} \leqslant c\|f\|_{L_{\infty}}
$$

for $\beta$ sufficiently large.

Proof. We may represent the operator on the left-hand side of (4.2) as

$$
\begin{aligned}
I-W+A-H+A_{\beta}-\Delta_{\beta}= & (I-W+A-H)(I-W)^{-1}\left(I-W+A_{\beta}\right) \\
& -(A-H)(I-W)^{-1} A_{\beta}-\Delta_{\beta} .
\end{aligned}
$$

In the appendix, we show that

$$
\left\|(A-H)(I-W)^{-1} A_{\beta}\right\| \rightarrow 0 \text { as } \beta \rightarrow \infty,
$$

and

$$
\left\|\Delta_{\beta}\right\| \rightarrow 0 \quad \text { as } \beta \rightarrow \infty .
$$

(Here, and in the following, the norm is the operator norm in the space $L_{\infty}$ ). The result then follows from (4.4), once we show that $(I-W+A-H)$ and $(I-W$ $+A_{\beta}$ ) have bounded inverses on $L_{\infty}$. But, from the hypotheses and Theorem 3.1, $I-W+A-H$ and $I-W+A$ have bounded inverses on $L_{\infty}$. Since the operator $A_{\beta}$ is related to $A$ by a simple translation, it follows that $I-W+A_{\beta}$ also has a bounded inverse on $L_{\infty}$, satisfying

$$
\left\|\left(I-W+A_{\beta}\right)^{-1}\right\|=\left\|(I-W+A)^{-1}\right\| .
$$

We can now apply the above result to obtain the required result for the finite-section equation. It is analogous to a result obtained by Anselone and Sloan $[1$, Theorem 10.1$]$ by entirely different methods.

THEOREM 4.2. Assume that condition (2.7) holds, and that neither

$$
\left(I^{+}-W^{+}-H^{+}\right) x^{+}=0, \quad x^{+} \in L_{\infty}^{+},
$$

nor

$$
\left(I^{-}-W^{-}\right) x^{-}=0, \quad x^{-} \in L_{\infty}^{-}
$$


has a nontrivial solution. Then for $\beta$ sufficiently large the finite-section equation (1.2) has a unique solution $y_{\beta} \in L_{\infty}(0, \beta)$ for each $f \in L_{\infty}(0, \beta)$. Moreover, there exists a constant $c>0$, independent of $\beta$ and $f$, such that

$$
\left\|v_{\beta}\right\|_{L_{\infty}(0, \beta)} \leqslant c\|f\|_{L_{\infty}(0, \beta)}
$$

for $\beta$ sufficiently large.

Proof. The hypotheses in the theorem are equivalent to those in Theorem 4.1. The result then follows from Theorem 4.1, on restricting attention in (4.2) to the interval $(0, \beta)$. (To obtain the estimate (4.8), set $f(t)=0$ for $t<0$ or $t>\beta$.)

It should be noted that an explicit expression can be given for the constant $c$ in Theorems 4.1 and 4.2-in fact from the argument used to prove Theorem 4.1, it is clear that we may take it to be

$$
c=(1+\varepsilon)\left\|\left(I+(I-W)^{-1} A\right)^{-1}\right\|\left\|(I-W+A-H)^{-1}\right\|,
$$

where $\varepsilon$ is any positive number. This is in marked contrast to the situation in [1], where the existence of a corresponding bound on the inverse of the finite-section operator is only inferred through a proof by contradiction based on the ArzelàAscoli theorem.

\section{Error Bounds}

In Section 2 we obtained results about the Wiener-Hopf equation (2.1) by incorporating it into an equation on the whole line, namely

$$
(I-W+A) z_{0}=f, \quad f \in L_{\infty},
$$

where the operator $A$ is compact on $L_{\infty}$. (In this section it is convenient to use the new notation $z_{0}$ for the solution of the extended Wiener-Hopf equation (5.1). The subscript zero reminds us that the discontinuity in the kernel and the solution occurs at the origin.) From an intuitive viewpoint, an essential difference between the compact operator $A$ and the noncompact operator $W$ is that $A$ is a 'localized' operator, with a kernel $a(t, s)$ that typically is small when either $t$ or $s$ departs far from the origin, while $W$ is an operator whose kernel depends only on the difference $t-s$, and which is therefore not localized at all. We might therefore expect that the solution of (5.1) would be similar to the solution of

$$
(I-W) z=f, \quad f \in L_{\infty},
$$

except near the origin. This is indeed the case, as is shown in the following lemma on the error $z_{0}-z$. 
LEMMA 5.1. Let condition (2.7) hold, and let $z \in L_{\infty}$ denote the unique solution of (5.2). Moreover, assume that (5.1) has the unique solution $z_{0} \in L_{\infty}$. Then

$$
\left|z_{0}(t)-z(t)\right| \leqslant[\gamma(t)+\gamma(t / 2)\|V\|+\eta(t / 2)\|W\|]\left\|z_{0}\right\|,
$$

where $V$ is as in Lemma 2.1, and

$$
\begin{aligned}
& \gamma(t)= \begin{cases}\int_{t}^{\infty}|w(s)| d s, & t>0, \\
\int_{-\infty}^{t}|w(s)| d s, & t<0,\end{cases} \\
& \eta(t)= \begin{cases}\int_{t}^{\infty}|v(s)| d s, & t>0, \\
\int_{-\infty}^{t}|v(s)| d s, & t<0 .\end{cases}
\end{aligned}
$$

Proof. It is easy to verify that

$$
z_{0}-z=-(I-W)^{-1} A z_{0}=-(I+V) A z_{0} .
$$

The result now follows from the definitions of $A$ and $V$, after application of some elementary inequalities.

We shall now combine the results of the previous section with Lemma 5.1, to obtain some insight into the behavior of the finite-section approximation when $\beta$ is large. If we denote by $o(1)$ any terms in an expression that vanish in norm as $\beta \rightarrow \infty$, then (4.4) may be written as

$$
\begin{aligned}
I-W+A-H+ & A_{\beta}-\Delta_{\beta} \\
& =(I-W+A-H)(I-W)^{-1}\left(I-W+A_{\beta}\right)+o(1) .
\end{aligned}
$$

From this it follows, for $\beta$ sufficiently large, that

$$
\begin{aligned}
\left(I-W+A-H+A_{\beta}-\Delta_{\beta}\right)^{-1} \\
=\left(I-W+A_{\beta}\right)^{-1}(I-W)(I-W+A-H)^{-1}+o(1) \\
=(I-W+A-H)^{-1}+\left(I-W+A_{\beta}\right)^{-1}-(I-W)^{-1} \\
\quad+\left(I-W+A_{\beta}\right)^{-1} A_{\beta}(I-W)^{-1}(A-H)(I-W+A-H)^{-1}+o(1) .
\end{aligned}
$$

It follows from an argument similar to that used in the appendix to prove (4.5) that

$$
\left\|A_{\beta}(I-W)^{-1}(A-H)\right\| \rightarrow 0 \text { as } \beta \rightarrow \infty,
$$


in consequence of which the fourth term of the above may be absorbed into the $o(1)$ term. Thus for $\beta$ sufficiently large we have

$$
\begin{aligned}
(I-W+A-H & \left.+A_{\beta}-\Delta_{\beta}\right)^{-1} \\
= & (I-W+A-H)^{-1} \\
& +\left(I-W+A_{\beta}\right)^{-1}-(I-W)^{-1}+o(1) .
\end{aligned}
$$

Now, let $y, y_{\beta}, z_{\beta} \in L_{\infty}$ be the (unique) solutions of

$$
\begin{gathered}
(I-W+A-H) y=f, \\
\left(I-W+A-H+A_{\beta}-\Delta_{\beta}\right) y_{\beta}=f,
\end{gathered}
$$

and

$$
\left(I-W+A_{\beta}\right) z_{\beta}=f
$$

respectively, where $f \in L_{\infty}$. Then from (5.3) we have

$$
y_{\beta}=y+z_{\beta}-z+o(1),
$$

where $z$ is the solution of (5.2).

Equation (5.4) has a simple interpretation, if we rewrite it as

$$
y_{\beta}-y=z_{\beta}-z+o(1),
$$

and restrict attention to the interval $(0, \beta)$. On this interval $y$ is the solution of (1.1), the full equation on the half-line, while $y_{\beta}$ is the solution of the finite-section equation (1.2). On the other hand $z$ is the solution of the equation

$$
z(t)-\int_{-\infty}^{\infty} w(t-s) z(s) d s=f(t),
$$

whereas, because $t<\beta, z_{\beta}$ is the solution of

$$
z_{\beta}(t)-\int_{-\infty}^{\beta} w(t-s) z_{\beta}(s) d s=f(t)
$$

That is, $z$ and $z_{\beta}$ satisfy the same equations as $y$ and $y_{\beta}$ respectively, except that the kernel $h(t, s)$ is absent, and secondly, the lower limit of integration is now $-\infty$ instead of 0 .

To the extent to which the approximation $y_{\beta}-y \approx z_{\beta}-z$ is valid (and it is valid uniformly for $\beta$ sufficiently large), the error $y_{\beta}-y$ is unaffected by the kernel $h(t, s)$, and shows no effects from the lower limit of integration. Rather, the error can be expected to be small over the entire interval $(0, \beta)$, except in the vicinity of the right-hand end.

To obtain a more precise result, we observe that an obvious extension of Lemma 5.1 yields

$$
\left|z_{\beta}(t)-z(t)\right| \leqslant\left[\gamma(t-\beta)+\gamma\left(\frac{t-\beta}{2}\right)\|V\|+\eta\left(\frac{t-\beta}{2}\right)\|W\|\right]\left\|z_{\beta}\right\|,
$$


where, with the aid of (4.7),

$$
\left\|z_{\beta}\right\| \leqslant\left\|(I-W+A)^{-1}\right\|\|f\| .
$$

Then from (5.5), together with Theorems 3.1 and 4.2, we obtain the error bound in the following theorem.

The constant $c_{1}$ in the theorem may be taken to be $\left\|(I-W+A)^{-1}\right\|$.

ThEOREM 5.2 Assume that condition (2.7) holds, and that neither

$$
\left(I^{+}-W^{+}-H^{+}\right) x^{+}=0, \quad x^{+} \in L_{\infty}^{+}
$$

nor

$$
\left(I^{-}-W^{-}\right) x^{-}=0, \quad x^{-} \in L_{\infty}^{-}
$$

has a nontrivial solution. Then for $\beta$ sufficiently large there exists $c_{1}$ independent of $\beta$ such that

$$
\begin{array}{r}
\left|y_{\beta}(t)-y(t)\right| \leqslant c_{1}\left[\gamma(t-\beta)+\gamma\left(\frac{t-\beta}{2}\right)\|V\|+\eta\left(\frac{t-\beta}{2}\right)\|W\|\right]\|f\|+\varepsilon_{\beta}, \\
0<t<\beta,
\end{array}
$$

where $y$ and $y_{\beta}$ are the solutions of (1.1) and (1.2), and $\varepsilon_{\beta} \rightarrow 0$ aS $\beta \rightarrow \infty$.

\section{Example}

A convenient illustration of the error bound in Theorem 5.2 is furnished by the integral equation

$$
y(t)-\frac{1}{\lambda} \int_{0}^{\infty} e^{-|t-s|} y(s) d s=1, \quad t \geqslant 0,
$$

for which both the exact solution $y$ and the finite-section approximation $y_{\beta}$ can be obtained analytically.

For this example $h(t, s) \equiv 0$ and

$$
w(t)=\frac{1}{\lambda} e^{-|t|} .
$$

Thus the Fourier transform $\hat{w}$ is

$$
\hat{w}(\nu)=\frac{2}{\lambda} \frac{1}{1+\nu^{2}},
$$

and therefore the condition (2.7) is satisfied provided $\lambda \notin[0,2]$. And in fact for $\lambda \notin[0,2]$ a solution $y \in L_{\infty}^{+}$of (6.1) exists and is unique (see [2]), and is given explicitly by

$$
y(t)=\frac{\lambda}{\lambda-2}-\frac{2}{(\lambda-2)(1+\mu)} e^{-\mu t}, \quad t \geqslant 0
$$


where

$$
\mu=\left(1-\frac{2}{\lambda}\right)^{1 / 2},
$$

with the positive branch of the square root to be taken for $\lambda \in \mathbb{R} \backslash[0,2]$.

It can easily be verified that for this example the integral operator $V$ defined in Lemma 2.1, with kernel $v(t-s)$, has $v$ given explicitly by

$$
v(t)=\frac{1}{\lambda \mu} e^{-\mu|t|} \text {. }
$$

Then one easily computes, using (2.6),

$$
\|W\| \leqslant \frac{2}{\lambda}, \quad\|V\| \leqslant \frac{2}{\lambda \mu^{2}}=\frac{2}{\lambda-2},
$$

and in a similar way the quantities $\gamma$ and $\eta$ defined in Lemma 5.1,

$$
\gamma(t)=\frac{1}{\lambda} e^{-|t|}, \quad \eta(t)=\frac{1}{\lambda-2} e^{-u|t|} .
$$

One also notes that for $\lambda \notin[0,2]$ equation (5.7) has no nontrivial solution, since otherwise (because $w$ is even, and $H^{+}=0$ ) there exists a nontrivial solution of (5.6), obtained by reflection in the origin.

Thus for this example Theorem 5.2 implies, for $\lambda \notin[0,2]$ and $\beta$ sufficiently large, the following bound on the error in $y_{\beta}$ :

$$
\begin{gathered}
\left|y_{\beta}(t)-y(t)\right| \leqslant c_{1}\left[\frac{1}{\lambda} e^{-(\beta-t)}+\frac{2}{\lambda(\lambda-2)}\left\{e^{-(\beta-t) / 2}+e^{-u(\beta-t) / 2}\right\}\right]+\varepsilon_{\beta}, \\
0<t<\beta,
\end{gathered}
$$

where $\varepsilon_{\beta} \rightarrow 0$ as $\beta \rightarrow \infty$.

If desired, an analytic expression for an upper bound on $c_{1}=\left\|(I-W+A)^{-1}\right\|$ can also be obtained for this example. One observes that for large $\beta$ the error is small for $\beta-t \gg \max (2,2 / \mu)$, i.e., for $t$ well away from the cut-off $\beta$; but that the effect of the cut-off is felt further and further away as $\mu \rightarrow 0$, i.e. as $\lambda \rightarrow 2$ from above.

It is interesting to compare this a priori bound with the exact error. For this example the exact solution of the finite-section approximation (1.2), obtained in the manner indicated in $[2, \S 3]$ and easily verified directly, is

$$
y_{\beta}(t)=\frac{\lambda}{\lambda-2}-\frac{2\left(e^{-\mu t}+e^{-\mu(\beta-t)}\right)}{(\lambda-2)\left(1+\mu+(1-\mu) e^{-\mu \beta}\right)}, \quad 0 \leqslant t \leqslant \beta .
$$

Thus the exact error on the interval $[0, \beta]$ is

$$
\begin{aligned}
y_{\beta}(T)-y(t)= & \frac{2}{(\lambda-2)\left(1+\mu+(1-\mu) e^{-\mu \beta}\right)} \\
& \times\left[-e^{-\mu(\beta-t)}+\left(\frac{1-\mu}{1+\mu}\right) e^{-\mu(\beta+t)}\right] .
\end{aligned}
$$


This is consistent with the bound (6.2), since the last term of (6.3) is bounded uniformly by

$$
\varepsilon_{\beta}:=\frac{2(1-\mu)}{(\lambda-2)(1+\mu)} e^{-\mu \beta},
$$

which converges to zero as $\beta \rightarrow \infty$.

\section{Concluding remarks}

We have considered the approximation of the integral equation (1.1) by the finite-section equation (1.2). By considering each of these equations as merely a limited view of a corresponding equation (with a discontinuous kernel) on the whole line, we have been able to recover known sufficient conditions for ensuring that (1.1) and (1.2) have unique, bounded solutions. Similar arguments have also allowed us to obtain a new asymptotic bound for the error of the finite-section approximation (see Theorem 5.2). The bound confirms the result of Anselone and Sloan [1], that $y_{\beta}(t) \rightarrow y(t)$ uniformly on every finite interval. It also establishes that for large $\beta$ the error is significant only in the vicinity of the cut-off $\beta$.

The finite-section equation (1.2) cannot in general be solved analytically, but can be solved numerically by any of the standard methods for integral equations of the second kind. However, a complete analysis of the numerical solution for (1.2) that takes into account the effect of varying $\beta$ is not yet available. It is expected that the techniques presented in the present paper will prove useful in such an analysis.

\section{Acknowledgment}

Ian Sloan acknowledges the support of the Australian Research Grants Scheme.

\section{Appendix}

In this appendix, we show that $\left\|\Delta_{\beta}\right\|$ and $\left\|(A-H)(I-W)^{-1} A_{\beta}\right\|$ converge to zero as $\beta \rightarrow \infty$, where the norms are the operator norms on $L_{\infty}$.

From the definition (4.3) of $\Delta_{\beta}$, it is easy to see that

$$
\begin{aligned}
\left\|\Delta_{\beta}\right\| \leqslant \max \left\{\int_{\beta}^{\infty} \mid\right. & w(s)\left|d s+\sup _{t>\beta} \int_{0}^{\infty}\right| h(t, s) \mid d s, \\
& \left.\times \sup _{t>0} \int_{\beta}^{\infty}|h(t, s)| d s, \int_{\beta}^{\infty}|w(-s)| d s\right\} .
\end{aligned}
$$


Because $w \in L_{1}$, it follows immediately that

$$
\int_{\beta}^{\infty}|w(s)| d s \rightarrow 0 \quad \text { as } \beta \rightarrow \infty
$$

and

$$
\int_{\beta}^{\infty}|w(-s)| d s \rightarrow 0 \quad \text { as } \beta \rightarrow \infty .
$$

Also, from assumption (1.5) we have

$$
\sup _{t>\beta} \int_{0}^{\infty}|h(t, s)| d s \rightarrow 0 \quad \text { as } \beta \rightarrow \infty .
$$

With slightly more difficulty we may prove in the following way that

$$
\sup _{t>0} \int_{\beta}^{\infty}|h(t, s)| d s \rightarrow 0 \text { as } \beta \rightarrow \infty \text {. }
$$

Letting

$$
\psi_{\beta}(t)=\int_{\beta}^{\infty}|h(t, s)| d s,
$$

it follows from (1.3) that for each $t \in[0, \infty)$ we have

$$
\psi_{\beta}(t) \rightarrow 0 \text { as } \beta \rightarrow \infty \text {. }
$$

Moreover, since

$$
\left|\psi_{\beta}\left(t^{\prime}\right)-\psi_{\beta}(t)\right| \leqslant \int_{0}^{\infty}\left|h\left(t^{\prime}, s\right)-h(t, s)\right| d s,
$$

it follows from (1.4) that the family $\left\{\psi_{\beta}\right\}_{\beta} \geqslant 0$ is equicontinuous at each point $t \in[0, \infty)$. Similarly, since

$$
\left|\psi_{\beta}(t)\right| \leqslant \int_{0}^{\infty}|h(t, s)| d s
$$

it follows from (1.5) that the family $\left\{\psi_{\beta}\right\}_{\beta} \geqslant 0$ is equiconvergent to zero as $t \rightarrow \infty$. Now let $t=x /(1-x)$, and define $\phi_{\beta} \in C[0,1]$ by $\phi_{\beta}(x)=\psi_{\beta}(t)$ for $0 \leqslant x<1$, $\phi_{\beta}(1)=0$. Then the family $\left\{\phi_{\beta}\right\}_{\beta \geqslant 0}$ is equicontinuous on $[0,1]$, and converges pointwise to zero as $\beta \rightarrow \infty$. Since pointwise convergence of an equicontinuous family on a compact set implies uniform convergence, it follows that

$$
\sup _{x \in[0,1]}\left|\phi_{\beta}(x)\right| \rightarrow 0 \quad \text { as } \beta \rightarrow \infty,
$$

which is equivalent to (Al). Thus,

$$
\left\|\Delta_{\beta}\right\| \rightarrow 0 \text { as } \beta \rightarrow \infty \text {. }
$$

To study the convergence of $\left\|(A-H)(I-W)^{-1} A_{\beta}\right\|$, note that it follows from Lemma 2.1 that

$$
(A-H)(I-W)^{-1} A_{\beta}=(A-H)(I+V) A_{\beta}=(A-H) A_{\beta}+(A-H) V A_{\beta} \text {. }
$$


From the definitions of $A, A_{\beta}, H$ and $V$ we obtain, by a straightforward but rather tedious calculation, the following bounds:

$$
\begin{gathered}
\left\|A A_{\beta}\right\| \leqslant 2\|W\| \int_{\beta / 2}^{\infty}|w(-s)| d s, \\
\left\|H A_{\beta}\right\| \leqslant\|H\| \int_{\beta / 2}^{\infty}|w(-s)| d s+\|W\| \sup _{t>0} \int_{\beta / 2}^{\infty}|h(t, s)| d s, \\
\left\|A V A_{\beta}\right\| \leqslant 2\|V\|\|W\| \int_{\beta / 3}^{\infty}|w(-s)| d s+\|W\|^{2} \int_{\beta / 3}^{\infty}|v(-s)| d s,
\end{gathered}
$$

and

$$
\begin{aligned}
\left\|H V A_{\beta}\right\| \leqslant & \|V\|\|H\| \int_{\beta / 3}^{\infty}|w(-s)| d s+\|W\|\|H\| \int_{\beta / 3}^{\infty}|v(-s)| d s \\
& +\|V\|\|W\| \sup _{t>0} \int_{\beta / 3}^{\infty}|h(t, s)| d s .
\end{aligned}
$$

Since $v \in L_{1}$, each of the above bounds converges to zero as $\beta \rightarrow \infty$. Thus $\left\|(A-H)(I-W)^{-1} A_{\beta}\right\| \rightarrow 0$ as $\beta \rightarrow \infty$.

\section{References}

[1] P. M. Anselone and I. H. Sloan, "Integral equations on the half-line", J. Integral Equations 9 (suppl.) (1985), 3-23.

[2] K. E. Atkinson, "The numerical solution of integral equations on the half-line," SIAM $J$. Numer. Anal. 6 (1969), 375-397.

[3] K. Jörgens, Linear integral operators (Pitman, Boston, 1982).

[4] M. A. Krasnosel'skii, "On a theorem of M. Riesz", Soviet Math. 1(1960), 229-231.

[5] M. G. Krein, "Integral equations on a half-line with kernel depending on the difference of the arguments", Amer. Math. Soc. Transl. (2) 22(1963), 163-288.

[6] I. H. Sloan and A. Spence, "Projection methods for integral equations on the half-line", IMA J. Numer. Anal., (to appear).

[7] N. Wiener, The Fourter integral and certain of its applications (University Press, Cambridge, 1933). 